\title{
A Gliding Arc Microreactor Power Supply System Based on Push-Pull Converter Topology
}

\author{
Piotr Krupski ${ }^{1, *}$ and Henryka Danuta Stryczewska ${ }^{2}$ (D) \\ 1 Department of Fundamentals of Technology, Lublin University of Technology, Nadbystrzycka 38, \\ 20-618 Lublin, Poland \\ 2 Department of Electrical Engineering and Electrotechnology, Lublin University of Technology, \\ Nadbystrzycka 38A, 20-618 Lublin, Poland; h.stryczewska@pollub.pl \\ * $\quad$ Correspondence: p.krupski@pollub.pl; Tel.: +48-85384673
}

Received: 16 April 2020; Accepted: 4 June 2020; Published: 9 June 2020

Featured Application: The publication is related to the properties of an atypical power supply. This source is used to supply two-electrode micro gliding arc discharge (GAD) reactors ( $\mu$ GAD). The principle consists in applying parasitic phenomena, whose parameters are limited in other power converters.

\begin{abstract}
The era of evolution in power electronic systems has led to a widespread displacement of power supplies operating at mains frequencies. Switched-mode power inverters offer possibilities incomparably higher than mains transformers. These trends have also entered into plasma technologies, including GlidArc plasma processing. The article presents the use of an inverter power supply for a miniaturized GlidArc. It is a demonstration of using a push-pull topology in an unusual application. A special part is devoted to parasitic phenomena in the inverter and the implementation of switching overvoltages as a way of improving the ignition parameters of the power supply. The results of the tests with a plasma reactor in air conditions as a process gas are also presented.
\end{abstract}

Keywords: non-thermal plasma; $\mu \mathrm{GAD}$; SMPS; push-pull topology

\section{Introduction}

A gliding arc discharge (GAD) plasma reactor is a special source of non-thermal plasma. The discharge begins in the ignition area, located above the process gas distribution nozzle. First, with the mains frequency power supply, a spark discharge appears, followed by an arc column, whose volume and length increases gradually due to the fast gas flow and electrodynamic forces. Limiting the arc column current and its cyclic sliding movement, the thermodynamic imbalance increases and the discharge becomes a glow type [1]. There is a significant imbalance of electron temperature and average gas temperature, due to which a source of high-energy electrons used in plasmochemical processes is obtained. Maintaining this temperature regime from the perspective of the plasma process itself is beneficial, due to both the thermal safety of materials subjected to plasma treatment using the GAD reactor and the low energy consumption of the process $[2,3]$.

There are widespread trends to be observed in the implementation of pulsed techniques for feeding non-thermal plasma reactors [1,4-6]. These are power supply systems for both single and multi-electrode GAD reactors. The GAD carried out by being forced with an alternating voltage of an average frequency of $10-20 \mathrm{kHz}$ has slightly different properties than the one with the sinusoidal excitation of main frequency [7]. Relatively short (millisecond) voltage periods do not allow the discharge to turn into a short-circuit arc, which makes it easier to maintain the state of thermodynamic 
imbalance than on the network frequency voltage. In addition, the pulse power source gives extensive options for controlling current and voltage parameters [3].

\section{Switched-Mode Power Supply}

The GAD plasma reactor is a non-linear energy receiver, hence the special requirements for switched-mode power supply (SMPS), which is used to supply non-thermal $\mu$ GAD discharges. First, the power supply must generate a high voltage, adapted primarily to the type of process gas and the electrodes' distance in the ignition zone, in order for the discharge in the process gas to ignite cyclically without additional ignition electrodes, initial ionization, and radiation. Secondly, it is beneficial for the power supply from the receiver's point of view to have the characteristics of a regulated power source. Therefore, the SMPS source should quickly and efficiently limit the discharge current, which subsequently maintains a stable glow discharge.

These SMPS properties have been achieved by combining many standard and custom energy conversion techniques in a pulse inverter. The use of a push-pull topology is common for converters increasing DC voltage by several dozen volts. Here, the push-pull inverter was used to take advantage of the parasitic phenomenon of commutation overvoltage, and thus improve the ignition parameters in $\mu G A D$. The push-pull topology is characterized by a complex primary winding with a tap in its half (two-section winding), to which the inverter's asymmetry is very sensitive. Despite the significant multiplication of voltages, making the primary winding is not complicated; it is much more difficult to design and make a high voltage secondary coil. Push-pull systems show good properties at high frequencies-comparable to full-bridge ones, which limits Miller's effect. However, they are rarely used to power digital electronics, because the switching surges arising in them require limitation and protection of the output stage, especially for sensitive microprocessor technology.

The use of full-bridge push-pull topology is non-standard, due to its properties. Assuming identical parameters of the supply voltage Uin, output voltage Uout, rated power $P n$, operating

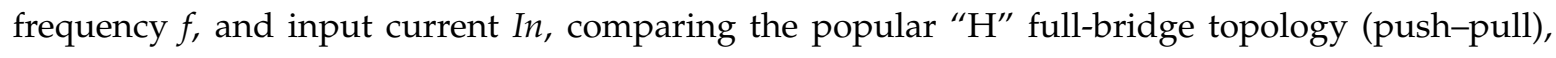
it should be assumed that, in a bridge converter, there are 4 fully controlled connectors and therefore inverter supply voltage $U i n$, inverter output voltage $U_{s}$, voltage on the primary winding $U p$, and voltage on the $U t$ controllable switches are equal to the supply voltage Uin, while the current of the switch $I t$ is equal to the input current $I n$, and the transformer ratio equals $N$.

In half-bridge push-pull topology, the output voltage of the inverter $U_{s}$ is equal to the supply voltage Uin. The voltage at the Ut controlled switch is doubled in the pulsing half-period, which is the result of the simultaneous sum of DC side voltages and the blocked primary winding voltage, which in turn is considered unfavorable in push-pull topology. The voltage on the full primary winding $U p$ is equal to doubled supply voltage $2 \mathrm{Uin}$, while the current of the controlled switch It is approximately equal to the input current $I n$, and the transformer ratio is $2 N$.

To describe the use of parasitic phenomena during energy conversion, a simplified diagram of this system is outlined in Figure 1. The input side of the power supply is standard for SMPS devices and has an input rectifier and filtering capacitors. There is also a power factor correction (PFC) compensator and a system for pre-charging capacitors. In this way, DC voltage is prepared to supply the push-pull topology converter. These are solutions that are treated as a standard in pulse technology and do not differ in this non-thermal plasma NTP power supply. Therefore, no details are referred to in this article.

As it can be seen in Figure 1, the positive pole of the filtered DC voltage is applied to the center tap in the primary coil. The 0 potential is connected alternately to the beginning and the end of the winding, according to the frequency and a pulse period. Executive valves are insulated gate bipolar transistor (IGBT). Switches designated as T1 and T2. Coils L2, L3, and L5 remain magnetically coupled in the substitute diagram. Coils L1, L4, and L6 represent leakage inductances.

The switching power supply is shown in Figure 2. 


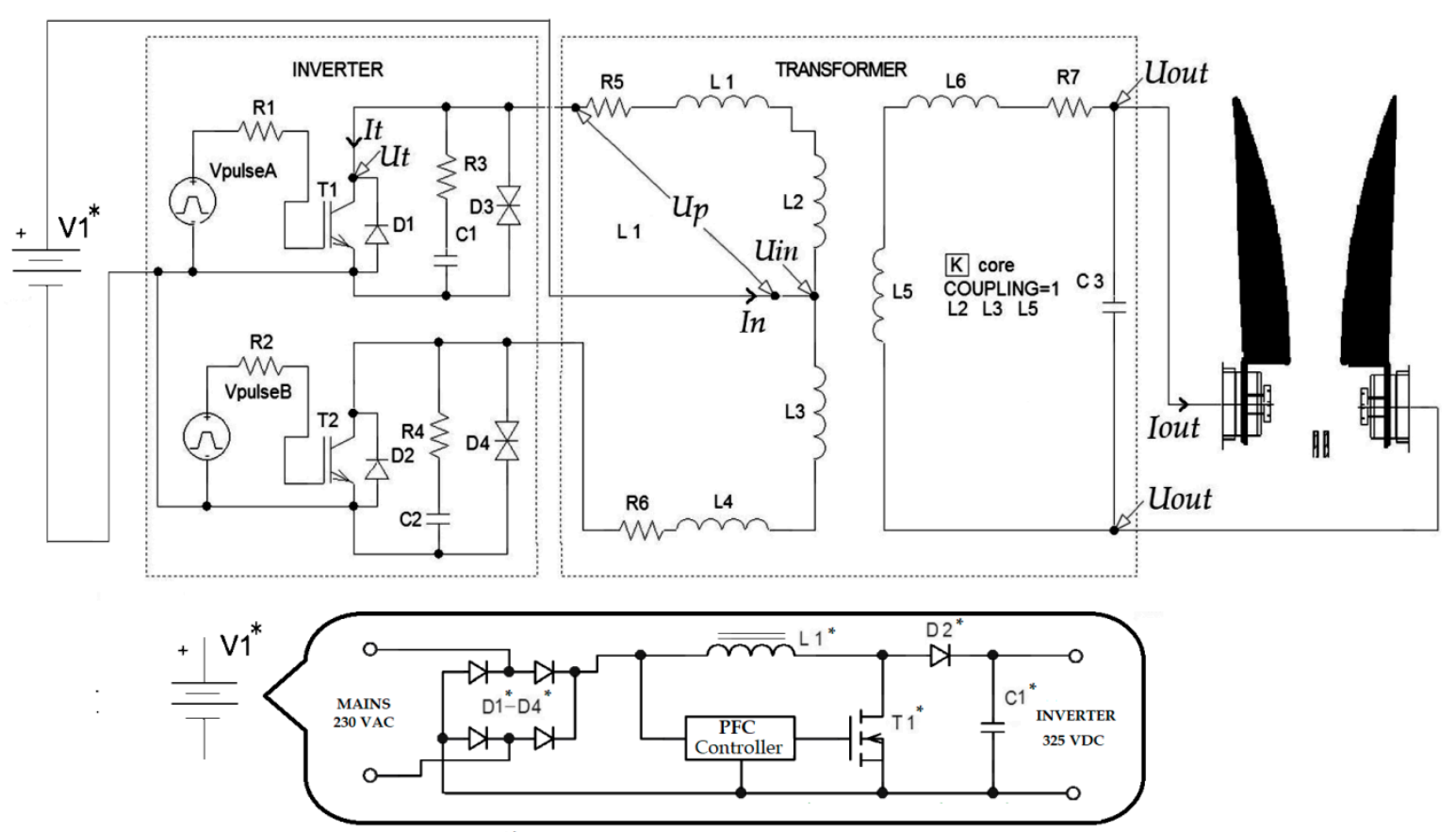

Figure 1. Simplified schematic diagram of the described processes of energy conversion in switched-mode push-pull power supply for micro gliding arc discharge reactors ( $\mu G A D)$.

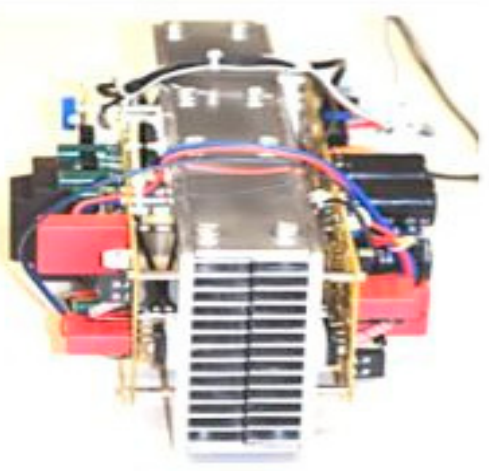

(a)

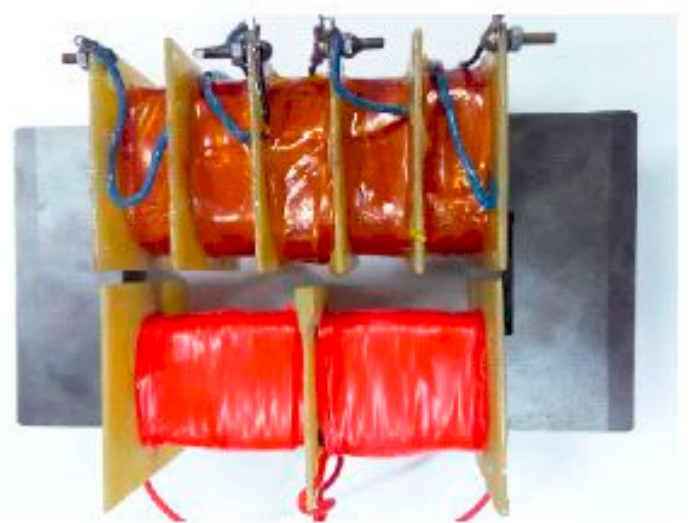

(b)

Figure 2. A photograph of the switched-mode power supply for $\mu \mathrm{GAD}$ inverter (a), and transformer (b).

During the pulse repetition, the fast-changing current is led through the rising edge to the magnetically coupled transformer coils, where the energy is transported to the secondary side via a magnetic flux. The fast current changes strongly depend on the leakage inductances. This is crucial due to the commutation overvoltages arising during the changes in current. Basically, they are used to generate high-voltage pulses (HVP) to support ignition of the plasma reactor. HVP can be used when the transformer's core was chosen in such a way as not to be a part of a low-pass filter for HVP. The voltage value of the switching overvoltage must be limited, due to the exposure of the switching elements in the primary current control system. That is why a special overvoltage control system was used. These are resistive-capacitive RC snubbers composed of elements R3, R4, and C1, C2. RC parameters require precise empirical selection. In addition, TRANSIL diodes (D3, D4) were introduced so that their speed and non-linearity would protect IGBTs. The system also has a secondary dissipation reactance and a C7 capacitor representing the parasitic capacity of the secondary side $[3,8,9]$. To see the non-standardity of the solution, it should be noted that typical push-pull power supplies attention is not being paid to obtain significant leakage inductances. The windings are placed on 
the $\mathrm{U}-\mathrm{U}$ core on separate columns here, to gain the appropriate characteristics and safety. This fact highly increases the scatter reactance. Under normal circumstances, they are limited as well as the resulting surges.

To be able to explain parasitic phenomena, the following simplifications can be made: It is necessary to isolate the resonance circuit for suppressed resonance, for example within the transistor T1. This circuit has $\mathrm{C} 1$ capacity and should be considered as the main one. In addition, there are parasitic capacitances (output of the $\mathrm{T} 1$ transistor, parasitic capacitances of the parallel diode, and transil diode). There is also parasitic capacitance within the primary coil. Inductance should be also indicated: main (L2), magnetically coupled in the transformer, and parasitic, marked as L1. Energy can be accumulated in both inductance as well as capacitance, and it is supplied when the transistor is turned on. Due to the fact that the principle of conservation of energy is obeyed, closing the transistor causes the energy to be redistributed repeatedly between inductance and capacitance. It is natural that, in this circuit, there is a coupled L2 coil that causes the transfer of surges to the secondary side. There are also attenuations and these are mainly resistances, including the deliberately introduced RC snubber resistance. Overvoltages were limited by the modulation of a current derivative and cut off with transil diodes. To give an idea of the quantitative parameters of the surge energy, there can be recalled the physical RC parameters of the selected snubber and the resistance of $22 \mathrm{ohms}$, and the capacity of several nanofarads. The overvoltage parameters are clearly visible in Figure 3.

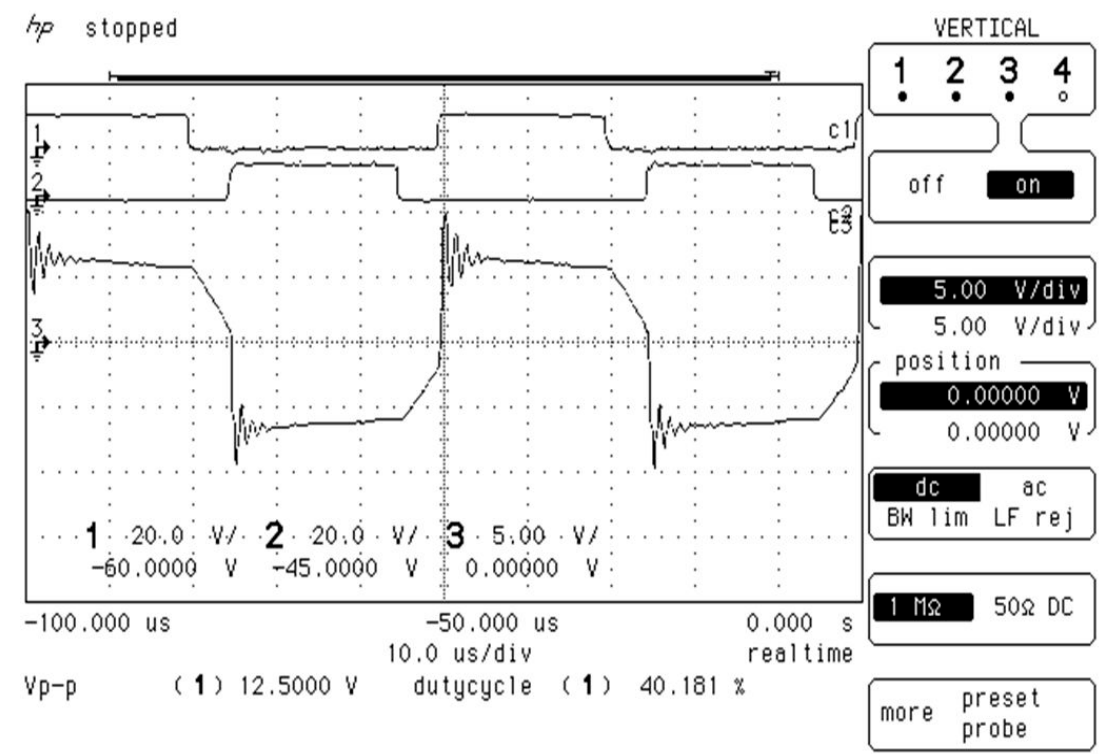

Figure 3. Electrical waveforms obtained in push-pull switched-mode power supply (SMPS), 1, 2-IGBT gate signals, 3-signal obtained in a single turn of secondary coil in no-load.

\section{Results}

The formation of commutation overvoltages can be seen in Figure 3.

Switching overvoltages (Figure 3) are observed in the idle state on a single turn of the secondary winding. The overvoltage is short-lived and has the form of suppressed resonance. Its occurrence on the secondary side greatly facilitates the ignition in the reactor, and when operating in air conditions as a process gas, completely eliminates the need for additional ignition systems.

The dynamic characteristics of the discharge in the ignition zone can be seen in Figure 4, where (a) shows the dynamic characteristics and (b) a photograph of the discharge. Figure 5 shows a photograph of a discharge in a $\mu$ GAD reactor for a long plasma cycle (about $11.5 \mathrm{~ms}$ ). Image $5 \mathrm{~b}$ shows the test using a fast camera and demonstrates the actual sliding movement of the discharge between two-knife electrodes. The discharges shown in Figure 5 are accompanied in each plasma cycle by decreasing current and increasing voltage waveforms-Figure 6. 


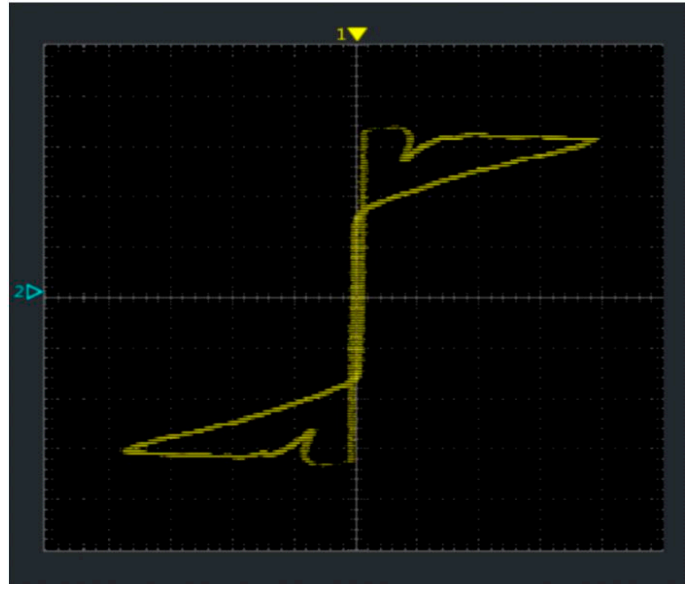

(a)

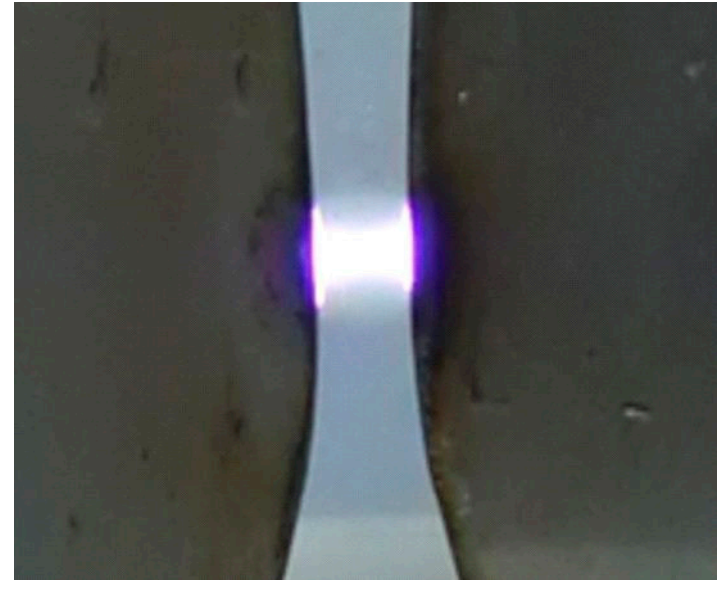

(b)

Figure 4. Discharge in the ignition space of the two-knife shaped electrodes of $\mu$ GAD under atmospheric pressure, (a) dynamic characteristics $i(t)=f[u(t)]$, where: signal 1: $u_{\mathrm{GA}}(t): 200 \mathrm{~V} / \mathrm{div}$, signal 2: $i_{\mathrm{GA}}(t) 50 \mathrm{~mA} /$ div, (b) discharge photo.

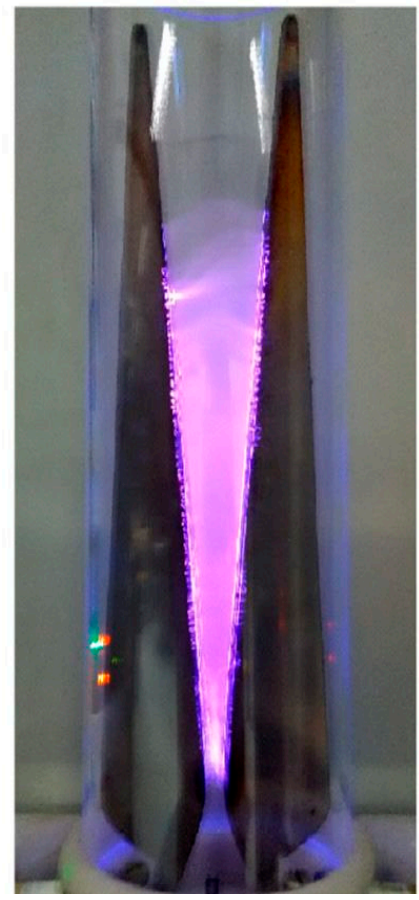

(a)

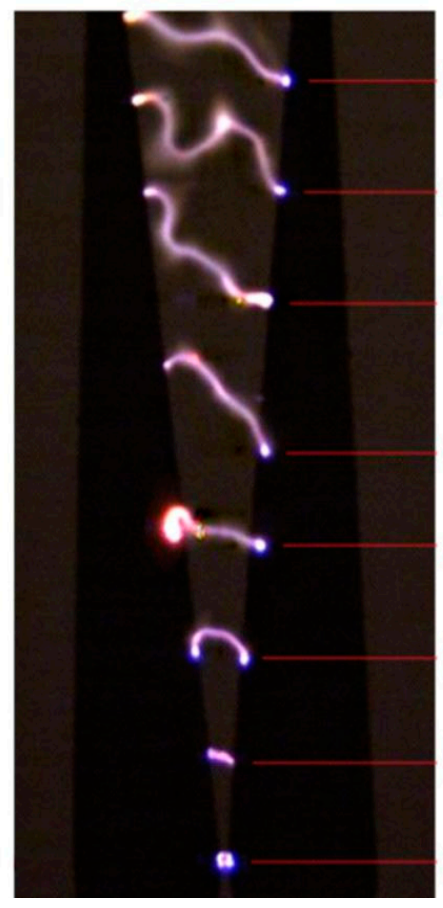

(b)

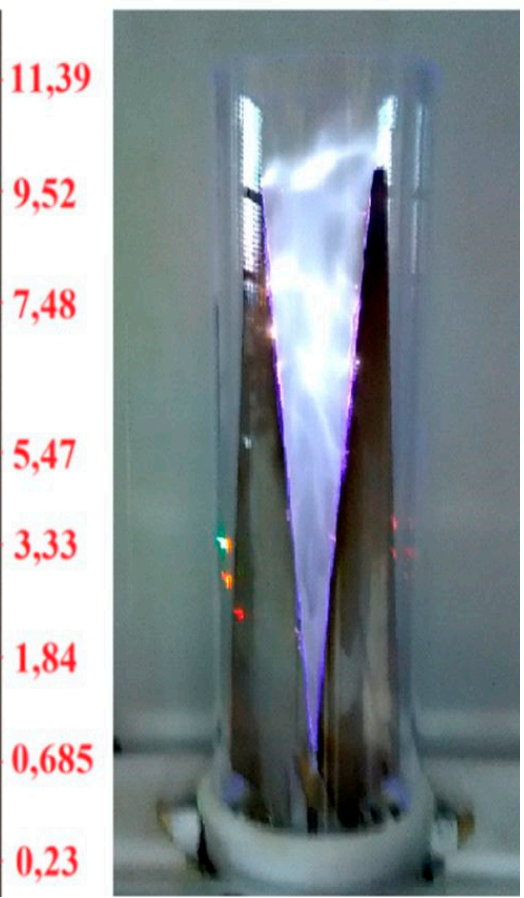

(c)

Figure 5. The discharge in the ignition space of two-knife shaped electrodes $\mu$ GAD under atmospheric pressure. Regular photos ((a)—discharge in helium, (c) - discharge in air); fast camera (b).

Figure 6 shows waveforms of the instantaneous voltage and current associated with a random plasma cycle (the discharge from the ignition zone to bursting at the electrode tips). 


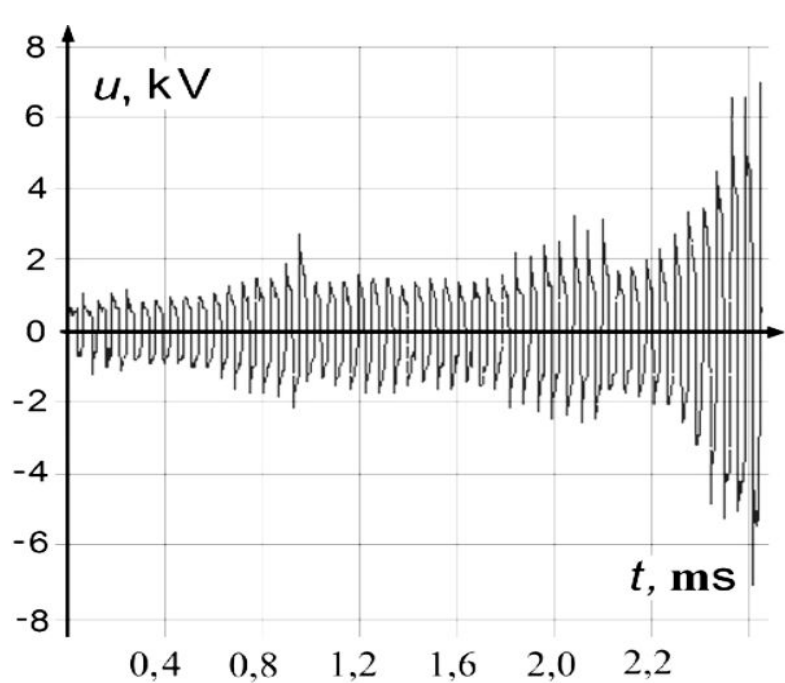

(a)

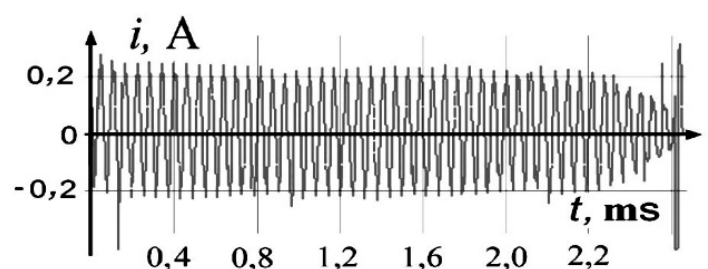

(b)

Figure 6. Electrical waveforms in the $\mu$ GAD reactor observed during a random plasma cycle under air conditions, voltage (a), and current $(\mathbf{b})$.

\section{Discussion}

Measurements and oscilloscopic studies show that waveforms in the plasma cycle are characterized by two periodicities. One of them results from the pulse frequency in SMPS and another one from the plasma cycle itself. About 50 pulse periods and a plasma cycle with a length of about $2.5 \mathrm{~ms}$ were observed on the waveforms. It was noted that in the plasma cycle, after ignition, the voltage reached a value of up to $0.5 \mathrm{kV}$. Then, the arc column moving up the electrodes lengthens and bends, reducing its conductance, and the voltage increases gradually, reaching $7 \mathrm{kV}$ at the tip of the electrodes. The reactor current changes the opposite way. After ignition, SMPS effectively limits the peak values at the level of $0.22 \mathrm{~A}$, and this value remained in the plasma cycle until the discharge conductance caused a current drop.

The discharge at the pulse-power source at $20 \mathrm{kHz}$ (Figure 5) from the beginning presents different features from the discharge at $50 \mathrm{~Hz}$. If the process gas stream is not fed through the nozzle, the discharge does not develop and moves up the electrodes, remaining in the ignition zone, and its voltage reaches hundreds of volts, due to the non-linearity of the receiver, which is the $\mu \mathrm{GAD}$ reactor's domain.

It is crucial how the traveling discharge changes its electrical parameters. With pulsed power supply and process gas distribution, observing the plasma reactor, one gets the impression that the discharge penetrates the inter-electrode space very closely. In noble gases, discharges are even more homogeneous. A photograph taken with an exposure time of $0.01 \mathrm{~s}$ does not allow observing the arc sliding over the knife electrodes. Only a fast camera test demonstrates the actual sliding movement of the discharge column along the knife electrodes.

\section{Conclusions}

On the basis of the research, it can be concluded that the non-standard push-pull topology may be successfully implemented in the power supply to conduct GADs. In addition, it should be said that the use of parasitic parameters in the form of leakage inductance allows an effective generation of ignition overvoltages. This, in turn, allows a $60 \%$ increase in voltage for GAD ignition.

It is also necessary to indicate some restrictions related to the selected topology of the power supply. If commutation overvoltages are used to improve ignition, use IGBTs with high maximum collector-emitter voltage. In this case, $\mathrm{U}_{\mathrm{CE}}=1.2 \mathrm{kV}$ keys were used. This is a high voltage, especially when compared to the full bridge topology, where it could be almost twice lower. 
The main pulse frequency band should be defined before the design stage. For the power supply described, it would not be possible to lower the frequency to $9 \mathrm{kHz}$, in the full pulse-width modulation (PWM) control range, because the core material and the winding do not allow it. The system has similar limitations at frequencies above $26 \mathrm{kHz}$. Changing the primary winding requires precise tuning of RC surge bands so that they only eliminate overvoltages that are dangerous to IGBT keys and at the same time allow the formation of ignition overvoltages. The use of microprocessor controllers, sensitive to radiated interference and conducted from the micro-gliding arc plasma reactor, also causes many difficulties [10]. These difficulties determined the use in the prototype of the power supply driver based on the analog, integrated PWM pulse width modulator.

The experience described in the article shows that appropriate modifications of push-pull topology, known for years, allow for the implementation of this atypical solution. Moreover, the main disadvantages of topology are used here as advantages. The power supply shows good characteristics and there is no need to use any additional ignition sources. This is very important especially in conditions of air as a plasma gas.

The designed and constructed power supply has the desired utility features required from the power electronic source of the plasma reactor. It also has numerous features that are its advantage over devices in which energy is transformed using a transformer with a network frequency, such as: good control parameters, power to volume ratio of the power supply, and high susceptibility to modifications.

\section{Patents}

1. Krupski, P.; Stryczewska, H. System and Method of Supplying a Plasma Reactor with a Gliding Arc Discharge. PL233,868B1, 31.12.2019 (https://api-ewyszukiwarka.pue.uprp.gov.pl/api/collection/ d100bb62cb87548cc9a19c392e177dd2\#search=\%22PL\%20233\%20868\%20B1\%22).

2. Diatczyk, J.; Pawłat, J.; Stryczewska, H.D.; Krupski, P.; Terebun, P.; Kwiatkowski, M. Microplasma Reactor with an Adjustable Distance between the Electrodes for Surface Treatment. PL 222,477, 29.07.2016. (https:/api-ewyszukiwarka.pue.uprp.gov.pl/api/collection/ 665450da4adc31070c7887a97be1cd16\#search=\%22\%20PL\%20222477\%20B1\%20\%22).

Author Contributions: Conceptualization, P.K. and H.D.S.; methodology, P.K.; software, P.K.; validation, H.D.S.; formal analysis, P.K.; investigation, P.K.; resources, H.D.S. and P.K.; writing-original draft preparation, P.K.; writing-review and editing, P.K. and H.D.S.; visualization, P.K.; supervision, H.D.S.; project administration, H.D.S.; funding acquisition, H.D.S. All authors have read and agreed to the published version of the manuscript.

Funding: This research received no external funding.

Conflicts of Interest: The authors declare no conflict of interest.

\section{References}

1. Jain, V.; Nigam, K.; Tanwani, N.; Adam, S.; Nimish, S.; Nema, S.K. Novel High Voltage Pulsing to Generate Uniform Glow Discharge Air Plasma for Environment Friendly Inline Treatment of Textile. In Proceedings of the 2019 IEEE Pulsed Power Plasma Science (PPPS), Orlando, FL, USA, 23-29 June 2019; pp. 1-3.

2. Komarzyniec, G.; Stryczewska, H.D.; Krupski, P. The Influence of the Architecture of the Power System on the Operational Parameters of the Glidarc Plasma Reactor. In Proceedings of the 2019 IEEE Pulsed Power Plasma Science (PPPS), Orlando, FL, USA, 23-29 June 2019; pp. 1-4.

3. Krupski, P.; Stryczewska, H.D.; Komarzyniec, G. The Push-Pull Plasma Power Supply-A Combining Technique for Increased Stability. In Proceedings of the 2019 IEEE Pulsed Power Plasma Science (PPPS), Orlando, FL, USA, 23-29 June 2019; pp. 1-4.

4. Itakura, K.; Kakemizu, H.; Nakaido, H.; Umetani, K.; Hiraki, E.; Ikenari, T.; Kawano, S. Soft-switching technique applicable to capacitive load for resonant inverter of plasma generator. In Proceedings of the IECON 2017-43rd Annual Conference of the IEEE Industrial Electronics Society, Beijing, China, 29 October-1 November 2017; pp. 1556-1562. 
5. Park, S.-H.; Sohn, Y.-H.; Cho, G.-H. SiC-Based 4 MHz 10 kW ZVS Inverter with Fast Resonance Frequency Tracking Control for High-Density Plasma Generators. IEEE Trans. Power Electron. 2020, 35, 3266-3275. [CrossRef]

6. Fang, Z.; Shi, Y.; Liu, F.; Zhou, R. Compact microsecond pulsed power generator driven by solar energy for dielectric barrier discharge applications. IEEE Trans. Dielectr. Electr. Insul. 2019, 26, 390-396. [CrossRef]

7. Aftyka, M.; Komarzyniec, G.; Stryczewska, H.D. Cooperation of the AC/DC/AC Power Converter with a GlidArc Multi-Electrode Plasma Reactor. In Proceedings of the 2018 Conference on Electrotechnology: Processes, Models, Control and Computer Science (EPMCCS), Kielce, Poland, 12-14 November 2018; pp. 1-4.

8. Krupski, P.; Stryczewska, H.D. The Investigation of the Properties of High-voltage Transformer in Nonthermal Plasma Pulse Power Supply. In Proceedings of the IEEE Explore-Post Conference Materials of 14th Selected Issues of Electrical Engineering and Electronics (WZEE) Conference, Szczecin, Poland, 19-21 November 2018; pp. 1-4.

9. Krupski, P.; Stryczewska, H.D. GLIDARC reactor power supply with ignition improvement. COMPEL-Int. J. Comput. Math. Electr. Electron. Eng. 2019, 38, 1274-1284. [CrossRef]

10. Mazurek, P. Selected methods to improve the electromagnetic compatibility of the plasma reactor. Przegląd Elektrotechniczny 2012, 88, 158-160.

(C) 2020 by the authors. Licensee MDPI, Basel, Switzerland. This article is an open access article distributed under the terms and conditions of the Creative Commons Attribution (CC BY) license (http://creativecommons.org/licenses/by/4.0/). 